\title{
Solving the Puzzle of Non-Reciprocity in Ultrafast Laser Writing
}

\author{
Aabid Patel ${ }^{1}$, Yuri Svirko ${ }^{2}$, Charles Durfee ${ }^{3}$, Rokas Drevinskas ${ }^{1}$, Peter G. Kazansky ${ }^{1}$ \\ 1. Optoelectronics Research Centre, University of Southampton, SO17 1BJ Southampton, United Kingdom \\ 2. Institute of Photonics, University of Eastern Finland, P.O. Box 111, FI-80101, Joensuu, Finland \\ 3. Department of Physics, Colorado School of Mines, 80401, Golden, Colorado, USA
}

The nanostructuring of transparent media with ultrashort laser pulses has attracted interest due to its unique applications. However, little is understood with respect to the physical mechanisms responsible for the peculiarities of the dielectrics inscribing with high intensity laser beams. It has been shown that spatio-temporal couplings (STC) inherent to the ultrashort pulses make inscribing sensitive to the writing direction [1,2] and that anisotropic photosensitivity [3] originates from the pulse front tilt (PFT). More recently, a greater appreciation of STC in focussing has been gained, shedding light on previously unknown parameters of the pulse such as a lighthouselike wavefront rotation [4] emphasizing the need for a better understanding of the STC effects in light-matter interaction. Nevertheless, understanding of the microscopic processes responsible for the modifications of dielectrics with ultrashort laser pulses and control of the writing process via STC is still lacking. Here we show with control of STC through the use of grating compressors, allows one to control and understand ultrafast phenomena associated with material modification. We reveal unambiguously that PFT gives rise to the nonreciprocity during femtosecond laser writing in transparent media and induces either an isotropic damage-like structure or a self-assembled nanostructure depending on the writing direction. This phenomenon is known as the "quill-writing effect" (Fig. 1a). A switching of the modification regime is observed when the translation of the beam is in the direction of the tilt, which can be qualitatively described in terms of a first-order phase transition.

(a)

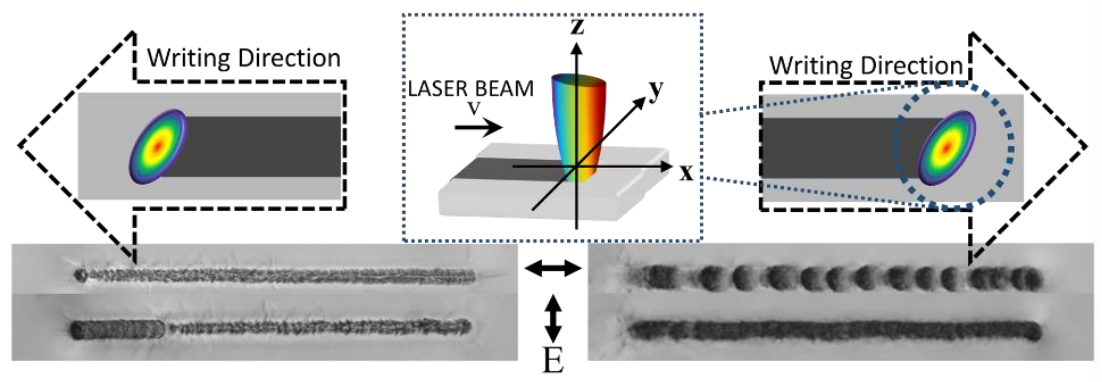

(b)

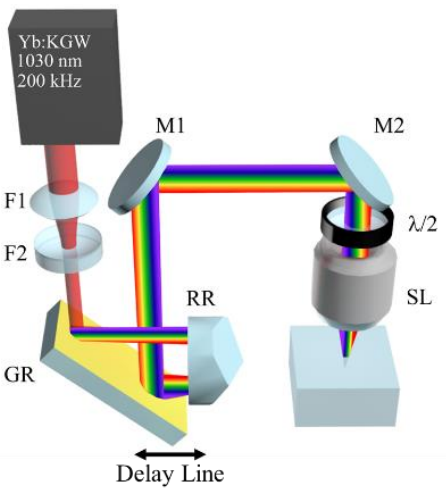

Fig. 1 (a) The quill-writing effect. Depending on the orientation of the PFT at the focus and its mutual orientation with the direction of the direct laser writing, different states of modification are observed; an isotropic damage-like structure or a self-assembled nanostructure. When translating the sample in the direction of the tilt, a transition from the damage-like state to the self-assembled nanograting is observed. (b) Sketch of the Spatio-Temporal Control set-up. The first grating compressor that controls temporal chirp is integrated with the $\mathrm{Yb}: \mathrm{KGW}$ laser system. The second compressor enables control of spatial chirp by changing the distance between the grating (GR) and a retroreflector (RR).

A regeneratively amplified, mode-locked Yb:KGW based femtosecond laser system (PHAROS, Light Conversion Ltd.) operating at $1030 \mathrm{~nm}$ with $200 \mathrm{kHz}$ repetition rate was used. Temporal chirp and spatial chirp were separately controlled with two grating compressors, allowing us to tailor the STC of the beam (Fig. 1b). The laser beam was focused $60 \mu \mathrm{m}$ into the bulk of fused silica with a $0.55 \mathrm{NA}$ aspheric singlet lens, mounted onto a XYZ linear air-bearing translation stage (Aerotech Ltd.), translating the sample at uniform speeds. The incoming laser light polarization was set either parallel or perpendicular to the spatial chirp with a $\lambda / 2$ waveplate. We have confirmed that the switching between modification regimes can be manipulated via the control of spatio-temporal couplings of the laser beam and that the non-reciprocal laser writing follows the direction of the PFT in the area where modification occurs. An understanding of these STC with complete control can introduce a new degree of freedom in ultrafast laser direct writing.

\section{References}

[1] W. Yang, P. G. Kazansky, Y. Shimotsuma, M. Sakakura, K. Miura, and K. Hirao, "Ultrashort-pulse laser calligraphy," Appl. Phys. Lett. 93, 171109 (2008).

[2] D. N. Vitek, E. Block, Y. Bellouard, D. E. Adams, S. Backus, D. Kleinfeld, C. G. Durfee, and J. A. Squier, "Spatio-temporally focused femtosecond laser pulses for nonreciprocal writing in optically transparent materials," Opt. Express 18, 24673-24678 (2010).

[3] P. G. Kazansky, Y. Shimotsuma, M. Sakakura, M. Beresna, M. Gecevičius, Y. Svirko, S. Akturk, J. Qiu, K. Miura, and K. Hirao, "Photosensitivity control of an isotropic medium through polarization of light pulses with tilted intensity front.," Opt. Express 19, 20657-64 (2011).

[4] H. Vincenti and F. Quéré, "Attosecond lighthouses: How to use spatiotemporally coupled light fields to generate isolated attosecond pulses," Phys. Rev. Lett. 108, 1-5 (2012). 